\title{
Filtration Efficiency of Bubble Scrubbers
}

\author{
$\underline{\text { R.D. Braddock }}^{\text {a }}$, R.A. Cropp ${ }^{\text {a }}$ and L. Boskovic ${ }^{b}$ \\ ${ }^{a}$ Griffith School of Environment, Griffith University, Gold Coast, Queensland \\ ${ }^{b}$ Queensland Institute of Business and Technology at Griffith University, Gold Coast, Queensland. \\ Email: R.Braddock@griffith.edu.au
}

\begin{abstract}
The passing of air through a column of water results in streams of bubbles rising through the chamber. Such a device has been used in science and industry, as a filtration mechanism to remove pollution particulates and gases from exhaust streams. Models of the individual processes inherent in the device have been developed over time. However, there are problems when attempting to match these theoretical results with the observations from experimental work that measures the efficiency of removal of the pollutants. There are some inconsistencies in determining the best operating conditions for the bubble chambers. For example, even researchers from the same research facility have provided inconsistent advice on the ideal operating conditions.
\end{abstract}

We use input-output analysis to model the bubble chamber as a complete system and incorporate existing individual process models as parts of the whole. The independent variables in the model are the water volume and the pollutant mass inside the scrubber chamber. The ensuing model is a set of two ordinary differential equations whose complexity depends on which individual process models are selected. The output processes include the "film" and "jet" droplets which are formed when the air bubble bursts at the water surface in the bubble chamber. Some of these droplets can be entrained and exit the chamber with the cleaned gases.

The equation for the water volume becomes redundant when the water volume in the chamber is held constant. Using the results from Fuchs, for particle absorption with in the column, and typical models for the generation of jet and film drops, the equation for the pollutant mass simplifies to a single linear ordinary differential equation. The outcomes are the existence of an equilibrium point and time varying exponential expressions for the efficiency of the chamber.

Measuring the efficiency of the scrubber is also compounded by the effects of measuring points and lengths and curvature of piping, and the expulsion of water droplets from the outlets. Some measurements of the deposition of particles in lengths of piping were made. Results show that the deposition depends on pipe length, curvature and flow rate. This effect needs to be accounted for in both the inlet and outlet piping and measurement points. Outgoing water droplets will also influence the size distribution and particle count at the outlet measuring point. The model suggests that the best way of measuring efficiency is to monitor the particle count in the water column, possibly by sampling and then taking a particle count in the sample. The sampling point should not be too close to the water surface.

The results provide guidance for operating the scrubber so that a desired efficiency is met including estimating time for maintenance. Monitoring the particle mass in the water is a better option than monitoring the input and outputs. Timing of the measurements is also important when performing experimental work and comparing with theoretical results, due to the time varying nature of the efficiency.

Keywords: Bubbles, nanoparticles, filtration efficiency 


\section{INTRODUCTION}

Industrial processes are becoming increasingly more complex in the quest for new methods and techniques in manufacturing, mining, power generation and coating of materials. These processes are increasingly focused on the use of nano-sized particles as modern science pushes the boundaries for new knowledge (Bemer et al. 2010). However, it has been shown that, for a given chemical composition and mass, the nanostructured particles could present a higher toxicity than micro size particles of the same type (Johnston et al. 2000). The emission of these nano-particles has health impacts as they can penetrate further into respiratory systems, and the emissions are being increasingly regulated (Oberdorster 2000).

Aerosols generated by winds passing over dry landscapes are also capable of carrying fine and ultrafine particles high into the atmosphere and then for considerable distances (Baddock et al. 2013). The study of dust as aerosols requires the collection of samples of the atmospheric nanoparticles of dust, so that their mineralogy and origins can be investigated. A similar problem arises in health studies, where virus samples need to be taken from human breath, so that the viruses can be identified (Agranovski et al. 2005).

Wet scrubbers and bubble chambers have been used for cleaning carrier gasses, and are receiving attention as possible collection devices for sampling atmospheric dust particles. Fibrous filters are more commonly used, but have a defect in that the pressure drop across the filter increases as the deposits of filtered particles build up. Eventually the filter fibres need to be cleaned or replaced. Filter unclogging may cause particles previously collected by the media to be put back into suspension and a deterioration of the filter structure, which may cause leaks and consequently a major decrease of filter efficiency. Filter clogging is most evident in metal coating processes because the particle concentrations are particularly high, $10^{8}$ to $10^{9} \mathrm{~cm}^{-3}$ and more than $90 \%$ of the emitted particles, by number, have a diameter smaller than $100 \mathrm{~nm}$. Also these fumes are highly clogging (Bemer et al. 2010).

As noted by Charvet et al. (2011) "there is still a lack of models able to predict the time evolution of the pressure drop and the life time of such filters...". One advantage of bubble processes and bubble chambers is that they operate at constant pressure drop, and do not require particle unloading..." Charvet et al. (2011) do note that the best operating conditions call for slow airflow rates, and higher water columns, and that there is a maximum penetration zone for particulates in the size range 10-30 nanometers. This is contrary to the experimental results of Cadavid-Rodriguez et al. (2014) which show that gas velocity does not greatly influence collection efficiency. Also the bubble columns do not put particles back into suspension because they collect ultrafine particles in the liquid phase (Cadavid-Rodriguez et al. 2014). The literature contains little on the separation efficiency of bubble column purification systems with respect to nanosize particles, as the most studies have focused on collecting large and micron size particles in bubble columns (Bandyopadhyay and Biswas 2006).

To the best of our knowledge only Koch and Weber (2012) and Charvet et al. (2011) and recently CadavidRodriguez et al. (2014) and Koch and Weber (2014) have studied the nanometric field. However, they note that further work is needed to generate optimal designs and operating conditions for the bubble filters for them to be able to viably compete with bag filters.

Several efficiencies of filtration by a bubble plant have been considered in the past. These include the theoretical efficiency obtained by applying Fuch's theory, and variations to this process model such as that of Pich and Shultz (1991), and a range of other improved models. Practical methods and experiments have been recently been performed by Charvet et al. (2011), by Koch and Weber (2012) and by Cadavid-Rodriguez et al. (2014) using bubble chambers and experimental arrangements to measure the particle concentrations before and after the bubble chamber. Charvet et al. (2011) show a "schematic view of the experimental setup" as do the others. In each case, piping is used to connect the bubble chamber to input and output measuring points. Charvet et al. (2011) used the Pich and Schultz model to compare with their experimental results, but the agreement between the theory and experimental results is not high.

Cosandey et al (2003) carried out a series of careful experiments and measured particle concentrations inside the water column, as well as above the water level but still inside the bubble chamber. In their Figure 11 they also show that the particles ejected from the bursting bubble are encapsulated in water. Charvet et al. (2011) did note that nanoparticles would deposit in pipes both upstream and downstream of the bubble chamber, and also on the walls of the chamber itself. They did take steps to measure the concentration downstream from a dry chamber and use that value as the input concentration in the actual experiments, to calculate the collection efficiency.

We have also performed similar experiments comparing experimental and model results, and have found it difficult to obtain good agreement. The experimental set up also may introduce unwanted effects due to 
varying lengths of tubing which carry the inflow of air and particles, and the out flow of gas and particles. There are other effects which need to be considered. We found it very difficult to improve on the agreement between experimental and theoretical results, as obtained by Charvet et al. (2011).

The aims of this paper are to examine the bubble chamber as an input/output device, and to pay particular attention to the particles which form the output stream. This will include the construction of a new system model of the bubble chamber, in an attempt to explain some of the discrepancies. This new model will focus more on the bubble chamber as an entity, rather than on the multitude of physical processes at play.

\section{EXPERIMENTAL RESULTS}

One aim of our experiments is to quantify the effects of the piping on the input flow of particles to the bubble chamber. The experimental setup followed that of Charvet et al. (2011) and will not be repeated here. In this research a glowing wire generator (GWG, Model 3709, AeroNanoTech,) was used to generate nickel (Ni) nano-particles. Nickel wire was placed between two electrodes and heated by electric current to produce a vapour in a quantity sufficient for super-saturation of the carrier gas. High purity compressed nitrogen gas, which was passed through a HEPA filter before reaching the GWG, was used as the carrier gas for the particles. The nanoparticles were formed due to mixing of hot $\mathrm{Ni}$ vapour with the cold nitrogen gas. The $\mathrm{Ni}$ wire was $0.5 \mathrm{~mm}$ in diameter and $5 \mathrm{~cm}$ long and the voltage used was $0.75 \mathrm{~V}$. Gas flow rates ranging from 1 to $5 \mathrm{l} / \mathrm{min}$ were used in the experiments.

The generation system can produce poly-disperse particles with diameters in the range $[5,30] \mathrm{nm}$. The characterisation of the particle size distribution is performed with a TSI Scanning Mobility Particle sizer, which measures the electrical mobility diameter of nanoparticles. To investigate the effects of the tubing on the input flow of particles to the bubble chamber tubing of three different lengths (1, 3 and $5 \mathrm{~m})$ was used to connect the GWG and the SMPS for subsequent measurement of particle concentration and size distribution. The aerosol stream was also forced through the empty chamber with a perforated aluminium sparger plate (150mm in diameter) with 31 orifii (300 $\mu \mathrm{m}$ orifice diameter).

A significant loss of particles occurred when the aerosol stream was passed through the empty sparger compared to the case when it travelled only a short length (1m) between the GWG and the SMPS (Figure 1). Increasing the tubing length from $1 \mathrm{~m}$ to $3 \mathrm{~m}$ increased the loss of the particles. However, the difference in particle concentration between 3 and $5 \mathrm{~m}$ long tubing was almost negligible, suggesting that the loss of the particles is occurring at the earlier stages. Measurements of the bubble diameters gave an average bubble size of $d_{B} \approx 2.5 \mathrm{~mm}$ and the number of bubbles $N_{B}$. The bubble diameter $d_{B}$, appears to be independent of the input air flux $Q_{\text {In }}$ for our setup while the number of bubbles produced is given by $N_{B}=6 Q_{I n} / \pi d_{B}^{3}$ bubbles per minute.

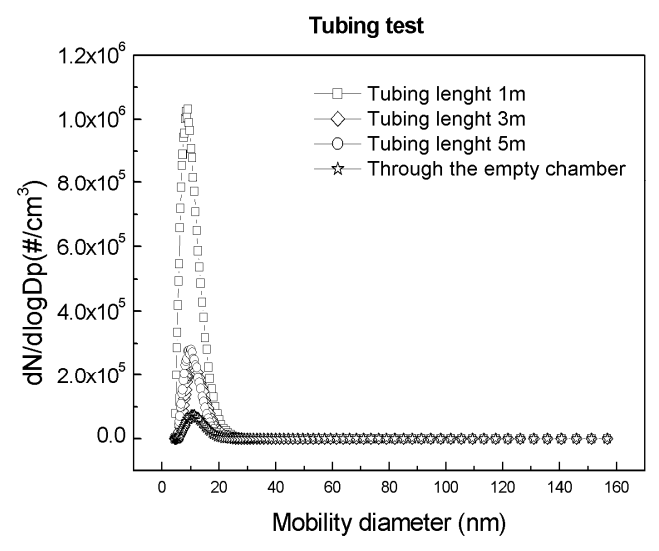

Figure 1. Effect of the tubing on the input flow of particles to the bubble chamber.

\section{THE FILM AND JET DROPS AS OUTPUT}

The essential features of a bubble chamber, the bubble cap and some notation, are shown in Figure 2. The volume of water in the bubble chamber at any time $t$ is $V(t)=A h(t)$ where the area of the chamber $A$ is constant. The concentration of particles in the input gas flow is given by $C_{P, I n}=M_{I n} / Q_{I n}$ (grams per litre). The deposition of aerosol particles in rising bubbles is described by the exponential deposition law, and after integrating and applying the boundary condition at the bottom of the tank, the concentration of particles at the top surface is:

$$
C_{P}(z=h)=C_{P-I n} e^{-\alpha h},
$$

where $C_{P}$ is the mass concentration of particles inside the bubbles, and $\alpha$ is the absorption coefficient. The form of the absorption coefficient was first studied by Fuchs and adjustments have been proposed over time (Charvet et al. 2011). The form used is immaterial to this paper and we use the forms contained in Charvet et 
al. (2011). Efficiency of filtration $\left(E_{F}\right)$ is traditionally taken in the form (Charvet et al. (2011), Koch and Weber (2012) and Cosandey et al. (2003)), where the concentrations can be experimental, or replaced by theoretical values;

$$
E_{F}=\frac{C_{P-I n}-C_{P}(z=h)}{C_{P-I n}},
$$

The mass of particles absorbed from a single bubble $\left(M_{P, B_{A}}\right)$ is then:

$$
M_{P, B_{A}}=V_{B}\left(C_{P-I n}-C_{P}(z=h)\right)=V_{B} C_{P-I n} E_{F} .
$$

where $V_{B}$ is the average volume of a bubble. The mass of particles still in the air in the bubble $\left(M_{P, B_{R}}\right)$, at the top surface is:

$$
M_{P, B_{R}}=V_{B} C_{P}(z=h)=V_{B} C_{P-I n}\left(1-E_{F}\right),
$$

and this will be expelled to the atmosphere above the water column when the bubble bursts.


Figure 2. The essential features of the Bubble Chamber (left) and the Bubble Cap (right). Notation for the Bubble Chamber (left) includes: $A$ the cross-sectional area of the bubble chamber, $h(t)$ the height of water in the chamber, $z$ the vertical dimension, $Q_{I n}$ the flux of air in and $M_{I n}$, the mass of particles in

the air in. Notation for the Bubble Cap (right) includes: $d_{B}$ the diameter of the bubble, $r_{F}$ the base

radius of the film, $\delta_{C}$ the thickness of the bubble film at rupture and $h_{C}$ the height of the cap.

Now consider the bursting bubble at the upper water surface. The bubble ejects air and particles not yet absorbed by the water. The bubble also ejects water and particles as film drops where the upper surface of the bubble ruptures and the enclosing water film is ejected (Cosandey et al. 2003). The thickness of the film at rupture $\left(\delta_{C}\right)$ is given by:

$$
\delta_{C}=0.5728 \frac{\sigma}{\rho_{W} c^{2}}\left(\frac{r_{B}^{2}}{r_{F}^{2}}\right),
$$

where $r_{F}$ is the base radius of the film, $c$ is the speed of sound in the gas, $\rho_{W}$ is the density of the water, $\sigma$ is the surface tension; and $r_{B}=d_{B} / 2$ is the radius of the bubble (Koch et al. 2000). Assuming that the bubble cap is spherical, and of uniform thickness, a simple integration gives the volume of the cap $\left(V_{F}\right)$ as 


$$
V_{F}=\delta_{C} 2 \pi r_{B}\left(r_{B}-\sqrt{r_{B}^{2}-r_{F}^{2}}\right),
$$

and this volume is ejected as the film bursts (Fig 2). When the bubble bursts, it emits droplets from the bottom part of the bubble as gravitation and surface tension forces produce a jet of water from the base of the bubble. The jet breaks down into a series of drops, "the jet drops", which are ejected from the surface. Reinke et al. (2001) discuss the size and number of the jet droplets, which are ejected. Their Figure 7 gives comparisons between experiments and calculations with respect to $d_{B}(\mathrm{~mm})$ and $d_{j d}(\mu \mathrm{m}$, the diameter of the jet drops). For the experimental setup described briefly in section 2 , above, then $d_{B}=2.5 \mathrm{~mm}$ and we can estimate a jet drop diameter $d_{j d}=400 \mu \mathrm{m}$. Using this relation we can estimate the volume then ejected $\left(V_{J D}\right)$ in the jet drops as:

$$
V_{J D}=N_{j d} \frac{4 \pi}{3}\left(\frac{d_{j d}}{2}\right)^{3} \approx 2 \frac{4 \pi}{3}(200 \mu \mathrm{m})^{3} .
$$

Hence, for our apparatus, the volume ejected in the film and jet drop per bubble is:

$$
V_{E}=V_{F}+V_{J D}=\delta_{C} 2 \pi r_{B}\left(r_{B}-\sqrt{r_{B}^{2}-r_{F}^{2}}\right)+2 \frac{4 \pi}{3}(200 \mu \mathrm{m})^{3}
$$

\section{THE BUBBLER AS AN INPUT-OUTPUT SYSTEM}

Now consider the bubbler as a whole and consider the input output model. Applying mass balance to the water in the bubbler $\frac{d V}{d t}=V_{I n}-N_{B} V_{E}$ where $V_{I n}$ is the flux of water into the bubbler, through the incoming gas flow, or as make-up water. Then $\frac{d V}{d t}=V_{I n}-\frac{Q_{I n} V_{E}}{V_{B}}$. Integrating yields:

$$
V(t)=V_{0}+t\left(V_{I n}-\frac{Q_{I n} V_{E}}{V_{B}}\right)
$$

where $V_{0}$ is the initial water content of the bubbler at $t=0$. The water content will remain constant provided that $V_{I n}=Q_{I n} V_{E} / V_{B}$ and the water make-up matches the water lost by means of the film and jet droplets. If $V_{I n}=0$ and no water make-up is available, then $V(t)$ will go to zero in time:

$$
t^{*}=\frac{V_{0} V_{B}}{Q_{I n} V_{E}},
$$

and the model is only valid for $0 \leq t<t^{*}$. Note that evaporative effects have not been included, but this is readily incorporated. Entrainment of droplets in the outgoing air stream is assumed to be $100 \%$ (i.e. no ejected droplets fall back to the water surface). This assumption will depend on the gas flow and the shape of the vessel and the exit area at the top. This can be modelled using a factor modifying the parameter ratio $C_{1}=V_{E} / V_{B}$.

\subsection{Particles in the Bubble Chamber}

Now consider the mass flux of particles in the bubble chamber, and assume that the water in the chamber and the particles are well mixed. This assumption is supported by the careful experiments of Koch and Weber (2012) who noted that "no significant change in the bubble and liquid velocities are observed over the whole width and height" in their bubble chamber. This conclusion is supported by the observational data given in their appendix. Cosandey et al. (2003) studied the movement of salts and particulates in a boiling pool and also undertook a careful examination of the distribution of the micron-sized particulates with height in the pool. Their results indicated that the particle concentrations "remain constant over the three sampling locations and no significant shift in the particle distribution is observed". However, they did note that this result could not be extrapolated to the air water interface due to the enrichment of this layer by ejected particles not being entrained into the exiting gas flow and falling back into the water, giving an enrichment of the particle concentration there. The "well mixed reactor" assumption will be made here. Now, the rate of change in the total mass of particles in the water column: 


$$
\frac{d M_{P}}{d t}=\text { flux in }- \text { flux out }=M_{I n}-M_{I n}\left(1-E_{F}\right)-C_{1} \frac{M_{P}(t)}{V(t)} Q_{I n}=M_{I n} E_{F}-C_{1} \frac{M_{P}(t)}{V(t)} Q_{I n},
$$

where it is assumed that the air is not absorbed into the reactor water and the fluxes of air in and out of the reactor are the same. Inclusion of this effect would require a further conservation equation for the air fraction. Compressibility effects have also been assumed to be small. This is a linear ordinary differential equation that is easily integrated numerically provided that $V(t)>0$. The boundary condition is taken in the form $M(t=0)=M_{0}$ where $M_{0}$, is the initial mass of particles in the bubbler. For the special case $V(t)=V_{0}$, a constant, then:

$$
\frac{d M_{P}}{d t}=M_{I n} E_{F}-\frac{C_{1} M_{P}(t)}{V_{0}} Q_{I n},
$$

and this equation has an equilibrium or steady state $M^{*}$, constant, where:

$$
M_{P}^{*}=\frac{M_{I n} E_{F} V_{0}}{C_{1} Q_{I n}},
$$

and represents the balance, at equilibrium, between the fluxes in and the fluxes out of the bubbler. A simple integration of (12) then gives:

$$
M_{P}(t)=M_{P}^{*}+\left(M_{0}-M_{P}^{*}\right) e^{-\left(\frac{C_{1}}{V_{0}} Q_{l n}\right) t} .
$$

The efficiency of the bubbler device $\left(E_{D}\right)$ is given by:

$$
E_{D}(t)=E_{F}-\frac{C_{1} Q_{I n}}{M_{I n} V_{0}} M_{P}(t),
$$

and this measures the efficiency just above the water level, and is time dependent. The half-life $t_{*}$ for the exponential decay is given by:

$$
t_{*}=\left(\frac{V}{C l Q}\right) \ln \left(2\left(1-\frac{M_{0}}{M_{P}}\right)\right)
$$

The half-life of the bubbler is enhanced by increasing $V_{0}$, the volume of the water, and by decreasing $Q_{I n}$, the air flow.

\section{DISCUSSION AND CONCLUSIONS}

Most experimental work in filtration uses forms of equation (2) to estimate the efficiency of the scrubber or filter. However, the precise location of the measuring positions, and the timing or scheduling of the experiments and measurements is rarely mentioned. The input-output model shows that the efficiency is dependent on the amount of filtered material, which has already been collected. Comparable results can only be guaranteed where the water in the scrubber is changed between experimental runs, and the measurements are made at comparable times on the passage to an equilibrium concentration.

The filtration efficiency question is further complicated by the experimental setup of the equipment, even down to the lengths of tubing carrying the gases and particles. This is clearly shown by the results in figure 1 where the efficiency of different lengths of tubing are reported without a scrubber attached. Where the tubing is curved inertial effects will also become important, more so for the larger particles. Note that the subsequent use of used or dirty tubing may result in the re-entrainment of particles deposited in the tubing during previous runs. The location of the input particle counter relative to the lengths of tubing will also be important. Charvet et al. (2011) appreciated these effects where they ran the equipment without water and used the output particle concentration as the effective input concentration for the wet experiments.

Measurement of the output concentration from a wet scrubber is even more complicated due to the presence of water from the film and jet drops. Indeed, the output measurements may well relate to the water particles, making a mockery of attempts to measure efficiency as a function of the input particle size. The situation is further complicated by the deposition of wet particles in the output tubing, where the presence of water will affect the deposition patterns. This has not been studied here. If precise experiments are to be conducted, then the experimental setup should include an evaporator on the outlet stream, with further deposition problems in the extra equipment. 
The model developed here has a major element missing, this being the flow regime on the scrubber, and above the water. Here, particles and water drops can return to the water column in the scrubber, and not be entrained in the output flow. The entrainment processes are also dependent on the air column above the water in the scrubber, a factor which needs to be considered as most experimental chambers are of fixed length, and increasing the water height necessarily decreases the length of the entrainment zone. Cosandey et al. (2003) appreciated the entrainment, non-entrainment, problem by attempting to use optical methods to measure particle concentrations just above the water in the scrubber. They also noted the enrichment of the upper surface water layer in the scrubber where particle concentrations can be up to 100 times the particle concentrations in the rest of the water column. Models do exist for this region, but are complicated by geometry and flow conditions, and are not considered here. However, they can be incorporated and will not detract from the simple message that the efficiency is time dependent.

The simple scrubber is a complicated device, and where precise measurements are to be made to investigate processes, the experiments need to be almost clinically clean and precise at each point in the equipment. The model so far developed, does provide some advice to operators of industrial scrubbers. Firstly, the concentration of particulates should be made from samples of water taken from the bulk of the water column. These measurements also need to be compared with the equilibrium particle concentration. This will enable the operator to adhere to output requirements for the particle scrubber. The Equilibrium level and performance requirements will also suggest the timing of such measurements to provide a sampling schedule.

\section{REFERENCES}

Agranovski, I. E., S. A. Safatov, A. I. Borodulin, O. V. Pyankov, V. A. Petrishchenko, A. N. Sergeev, V. Agranovski, and S. A. Grinshpun. 2005. New personal sampler for viable airborne viruses: feasibility study. . Journal of Aerosol Science 36:609-617.

Baddock, M., L. Boskovic, C. Strong, G. McTainsh, J. Bullard, I. Agranovski, and R. A. Cropp. 2013. Ironrich nanoparticles formed by Aeolian abrasion of desert dune sand. . Geochemistry, Geophysics, Geosystems. 14:3720-3729.

Bandyopadhyay, A., and M. N. Biswas. 2006. Fly-ash scrubbing in a tapered bubble column scrubber. Process Safety and Environmental Protection 84:54-62.

Bemer, D., R. Regnier, I. Subra, B. Sutter, M. T. Lecler, and Y. Morele. 2010. Ultrafine particles emitted by flame and electric arc guns for thermal spraying of metals. Annals of Occupational Hygiene 54:607614.

Cadavid-Rodriguez, M. C., A. Charvet, D. Bemer, and D. Thomas. 2014. Optimization of bubble column performance for nanoparticle collection. Journal of Hazardous Materials 271:24-32.

Charvet, A., N. Bardin-Monnier, and D. Thomas. 2011. Can bubble columns be an alternative to fibrous filters for nanoparticles collection? Journal of Hazardous Materials 195:432-439.

Cosandey, J. O., A. Gunther, and P. R. von Rohr. 2003. Transport of salts and micron-sized particles entrained from a boiling water pool. Experimental Thermal and Fluid Science 27:877-889.

Johnston, C. J., J. N. Finkelstein, P. Mercer, N. Corson, R. Gelein, and G. Oberdorster. 2000. Pulmonary effects induced by ultrafine PTFE particles. Toxicology and Applied Pharmacology 168:208-215.

Koch, D., and A. P. Weber. 2012. Separation of gas-borne nanoparticles in bubble columns. Journal of Aerosol Science 53:61-75.

Koch, D., and A. P. Weber. 2014. Stabile nanosuspensionen durch die abscheidung von gasgetragenen wirkstoff-nanopartikeln in blasensaulen. Chemical Engineering Technology 86:280-286.

Koch, M. K., A. Vosnacke, J. Starflinger, W. Schultz, and H. Unger. 2000. Radionuclide Re-entrainment at Bubbling Water Pool Surfaces. Journal of Aerosol Science 31:1015-1028.

Oberdorster, G. 2000. Pulmonary effects of inhaled ultrafine particles. International Archives of Occupational and Environmental Health 74:1-8.

Pich, J., and W. Shultz. 1991. On the theory of particle deposition in rising gas bubbles; the absorption minimum. Journal of Aerosol Science 22:262-272.

Reinke, N., A. Vosnacke, W. Schutz, M. K. Koch, and H. Unger. 2001. Aerosol generation by bubble collapse at ocean surfaces. Water, Air, and Soil Pollution 1:333-340. 